\title{
Activity as the Main Explanation of Light Variations of $o$ And - Towards a Rotational Modulation Model
}

\author{
J.P. Sareyan ${ }^{1}$, J. Chauville ${ }^{2}$, M. Alvarez ${ }^{3}$, G. Guerrero ${ }^{4}$, A. Arellano \\ Ferro $^{5}$, P. Mathias ${ }^{1}$, J. Peña ${ }^{5}$ \\ ${ }^{1}$ Observatoire de la Côte d'Azur, Nice, France; ${ }^{2}$ Observatoire de \\ Meudon, France; ${ }^{3}$ Observatorio Astronómico Nacional. UNAM. \\ Ensenada B.C. México; ${ }^{4}$ Osservatorio di Milano Merate, Italy; \\ ${ }^{5}$ Instituto de Astronomía. UNAM, México D.F. México.
}

Observations carried out for 5 years on $o$ And show that 65 to $85 \%$ of its light variations can be described by a double wave: $A \sin \left(2 \pi t / P_{1}+\phi_{1}\right)+$ $B \sin \left(2 \pi t / P_{2}+\phi_{2}\right)+C$ with $P_{1} \approx 1.6 \mathrm{~d}=2 P_{2}$. When determined independently, $P_{1}$ and $P_{2}$ are always found in a 2:1 ratio (within $1 \%$ ), while they can vary together by as much as $4 \%$. The peak to peak amplitudes of this doublewave fit lay between 40 and $140 \mathrm{mmag}$ (and can even be reduced to less than 10 mmag - our 1987 observations). The rest of the light variations do not show any permanent period or behavior, although a $\sim 2.3 \mathrm{~d}$. (i.e. $\approx 3 \mathrm{P}_{1} / 2$ ) period is frequently detected. Sometimes a marginal $\sim 6$ d. period or time constant has been detected.

In spite of the quality of our photometric data, the precision on the periods and amplitudes obtained over a few nights is never increased by longer observations: our phase diagrams show significant irregular displacements around the average double-wave analytical solution if we include longer data strings (Fig. 1). This phenomenon was already apparent in our 1992 study (Sareyan et al., 1998): the star shows real irregular behaviour superimposed onto its double-wave "mean" light curve; these changes may show up as a progressive, or sometimes abrupt, modification of the shape of the double-wave light curve (Fig. 1).

The $\mathrm{B} / \mathrm{A}$ ratio of amplitudes can vary from $\sim 0.34$ to 2.8 and the $(\mathrm{A}+\mathrm{B})$ sum from 18 to $70 \mathrm{mmag}$ ( $7 \mathrm{mmag}$ in 1987), so that in a pulsational hypothesis the total available energy should vary by a factor close to 100 , and the energy transfer between $P_{1}$ and $P_{2}$ should reach a factor 350 during our 5 years campaign. Thus, as far as regular behaviour is concerned, it does not seem that the multiperiodicity found in $o$ And $(\mathrm{P}, \mathrm{P} / 2$, possibly $3 \mathrm{P} / 2$, and in some occasions a $\sim 6 \mathrm{~d}$ period or time constant) could be related to any known model of pulsation.

As the period $\mathrm{P}_{1}(1.6 \mathrm{~d})$ is the likely rotation period of the principal (B5 II-IIIe) star of $o$ And, a possible model that can reproduce both the $1.6 \mathrm{~d}$ doublewave period and its significant irregularities is that of two rather large zones of activity linked to the star's photosphere, and roughly opposite in longitude, as shown by the permanent (1975 through 1998), but variable, double-wave light curves. The total area of these active regions, and/or their "intensity" projected along the line of sight, varies very much during a decade, which seems to be their characteristic time scale. On the apparent borders or on the sides of these active regions, abrupt changes of the star activity occur on a time scale of a few days or less, which shifts the "gravity center" of the light curves extrema. These short 

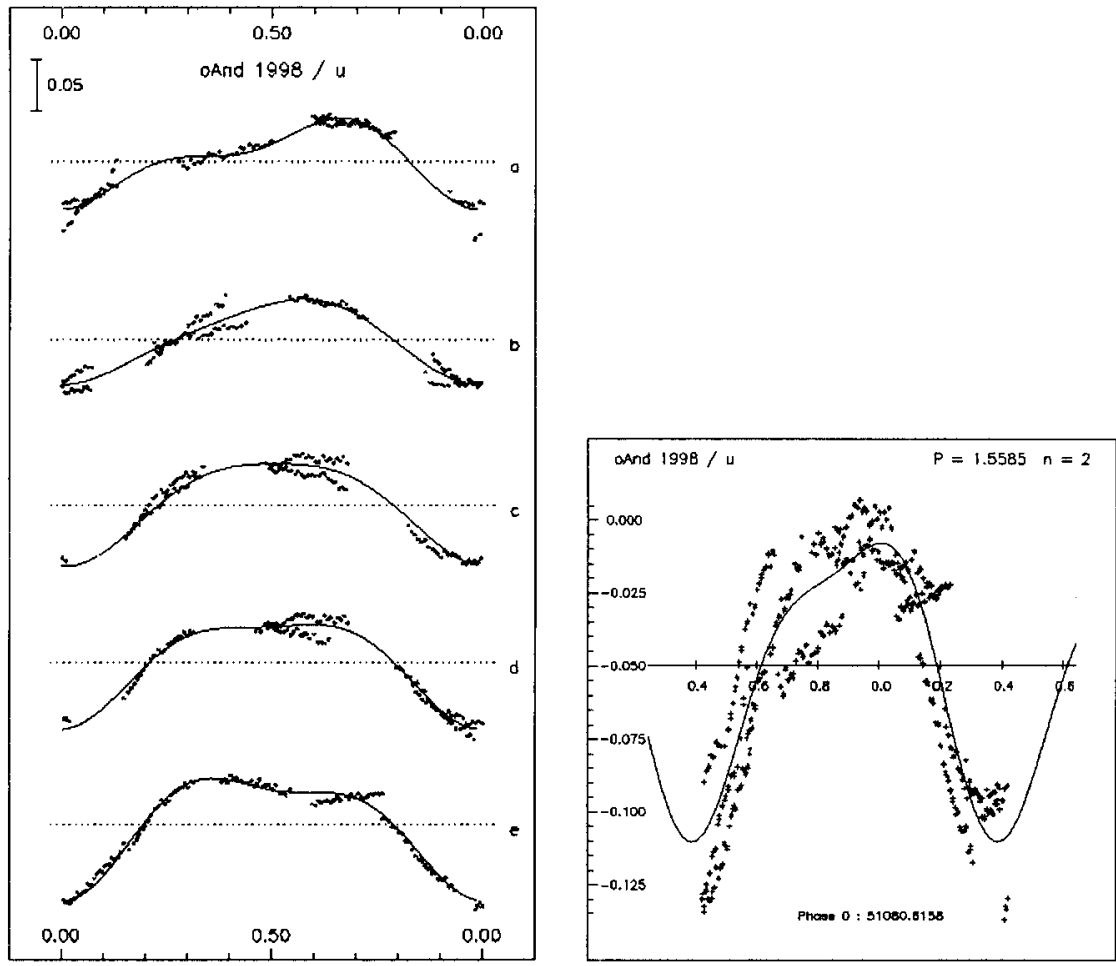

Figure 1. Left: the $1998 u$ data as an example of the simple least squares fit with a common $1.5585 \mathrm{~d}$ double-wave period (13 contiguous nights). Note the irregular displacements around this mean fit. Right: same $1998 u$ data fit with a five nights sliding average.

time scale localized events account for the short time scale perturbations of the observed amplitudes and apparent modulation of the rotation period.

The eventual longer time scale $(\sim 6 \mathrm{~d})$ could be an overmodulation due to changes in the opacity of the envelope.

Acknowledgments. Much of this work has received the continuous support of the CNRS, France and the CONACYT and UNAM, México.

\section{References}

Sareyan, J.P., et al. 1998, A\&A, 332, 155 